\title{
Analysis of radioactive implant migration in patients treated with iodine-125 seeds for permanent prostate brachytherapy with MRI-classified median lobe hyperplasia
}

\author{
Koichiro Muraki, MD', Chikayuki Hattori, MD!, Etsuyo Ogo, MD, PhD', Hiroaki Suefuji, MD, PhD', Hidehiro Eto, MD', \\ Chiyoko Tsuji, MD', Yusaku Miyata, MD', Toshi Abe, MD, PhD', Katsuaki Chikui, MD², Makoto Nakiri, MD, PhD², \\ Tsukasa Igawa, MD, PhD², Tatsuyuki Kakuma, PhD, MPH ${ }^{3}$ \\ 'Department of Radiology, Kurume University, Japan, ${ }^{2}$ Department of Urology, Kurume University, Japan, ${ }^{3}$ Biostatistics Center, \\ Kurume University, Japan
}

\begin{abstract}
Purpose: Prostate cancer with median lobe hyperplasia (MLH) is a relative contraindication for permanent prostate brachytherapy (PPB) because of an increased risk of post-implant dysuria and technical difficulties associated with achieving stability while implanting within the intravesical tissue. We examined treatment outcome, seed migration, and urination disorders after treatment in MLH patients in order to determine to what degree MLH implants could be stabilized.

Material and methods: Between March 2007 and December 2016, 32 patients had MLH identified radiologically on magnetic resonance imaging, and 193 patients did not have MLH (non-MLH). All patients were treated with loose seeds. In this study, seed migration was defined as a seed distant from the target $(\geq 1.5 \mathrm{~cm})$ and/or with no dosimetric contribution to the target. The MLH patients were divided into $2 \mathrm{MLH}$ groups of mild $(<10 \mathrm{~mm})$ and severe $(\geq 10 \mathrm{~mm}$ ) MLH by measuring the distance between the posterior transitional zone and the prostatic tissue protruding into the bladder. We retrospectively analyzed seed migration, dose-volume histograms (DVH), and genitourinary toxicity.

Results: MLH was classified as mild in 24 patients and severe in 8. Seed migration occurred in 61 (31.6\%) of 193 non-MLH patients and 10 (31.5\%) of 32 MLH patients. Implant seed migration and low-dose level of median lobe tended to be high in severe MLH cases. International Prostate Symptom Score (IPSS) peaked one month after implantation, but then resolved slowly and returned to around the pre-treatment level after one year. There were no severe complications.

Conclusions: MLH does not appear to be a strong contraindication for low-dose-rate brachytherapy. However, we found that the seed migration and degree of cold spots tended to be higher in severe MLH cases than in others; therefore, close attention when treating severe MLH cases must be paid.

Key words: prostate cancer, median lobe hyperplasia, LDR brachytherapy, seed migration.

\section{Purpose}

Patient selection criteria for low-dose-rate brachytherapy (LDR-BT) according to the American Brachytherapy Society (ABS) and GEC-ESTRO have been described. The ABS Prostate Low-Dose-Rate Task Group specified that substantial median lobe hyperplasia (MLH) is a relative contraindication for LDR-BT due to an increased risk of post-implant dysuria (urinary retention, acute residual urine, and hematuria), and becomes a bottleneck for technical difficulties in achieving stabil- ity when performing implantation in intravesical prostatic protrusion (IPP) with consideration of a bladder neck dose $[1,2]$.

However, the threshold levels regarding the degree of IPP as a contraindication and the magnetic resonance imaging (MRI) findings depicting MLH are not clearly described in the ABS criteria.

A median lobe of prostate tissue can increase bladder outlet resistance by causing a 'valve ball' type of bladder outlet obstruction (BOO), with incomplete opening 
of the bladder neck and disruption of its funneling effect [3]. Previous studies have reported that a prostatic mass with greater protrusion involves more severe voiding dysfunction by causing more serious BOO [4-6]. A systematic review of the overall literature reported that five studies used a threshold IPP of $10 \mathrm{~mm}$ to define BOO [7].

In this study, we verified our assumption that it is difficult to achieve stability while implanting within the intravesical prostatic protrusion without dysuria in a severe MLH group ( $\geq 10 \mathrm{~mm}$ ). We divided patients into two groups based on the degree of MLH: the severe MLH group ( $\geq 10 \mathrm{~mm}$ ) and the non-severe MLH group $(<10 \mathrm{~mm})$. Clinical data, dose-volume histograms (DVH), and seed migration of the two groups were analyzed to define the clinical significance of MLH.

\section{Material and methods}

We evaluated the distance of median lobe hyperplasia (dMLH) objectively on sagittal MRI using Wallner technique with trans-rectal ultrasound (TRUS) (Figure 1) [8]. The treatment outcomes, seed migration, and urinary disorders after the treatment in MLH patients who received LDR-BT were examined. Also, the indications of LDR-BT, planning, and technical issues as well as toxicity and efficacy for MLH were discussed. Additionally, the degree to which MLH implants could be safely stabilized was explored. Cases, in which the degree of MLH was $>20 \mathrm{~mm}$ due to concerns in relation to a high-dose at the bladder neck were excluded; such patients are offered a resection or holmium laser enucleation of the prostate (HoLEP).
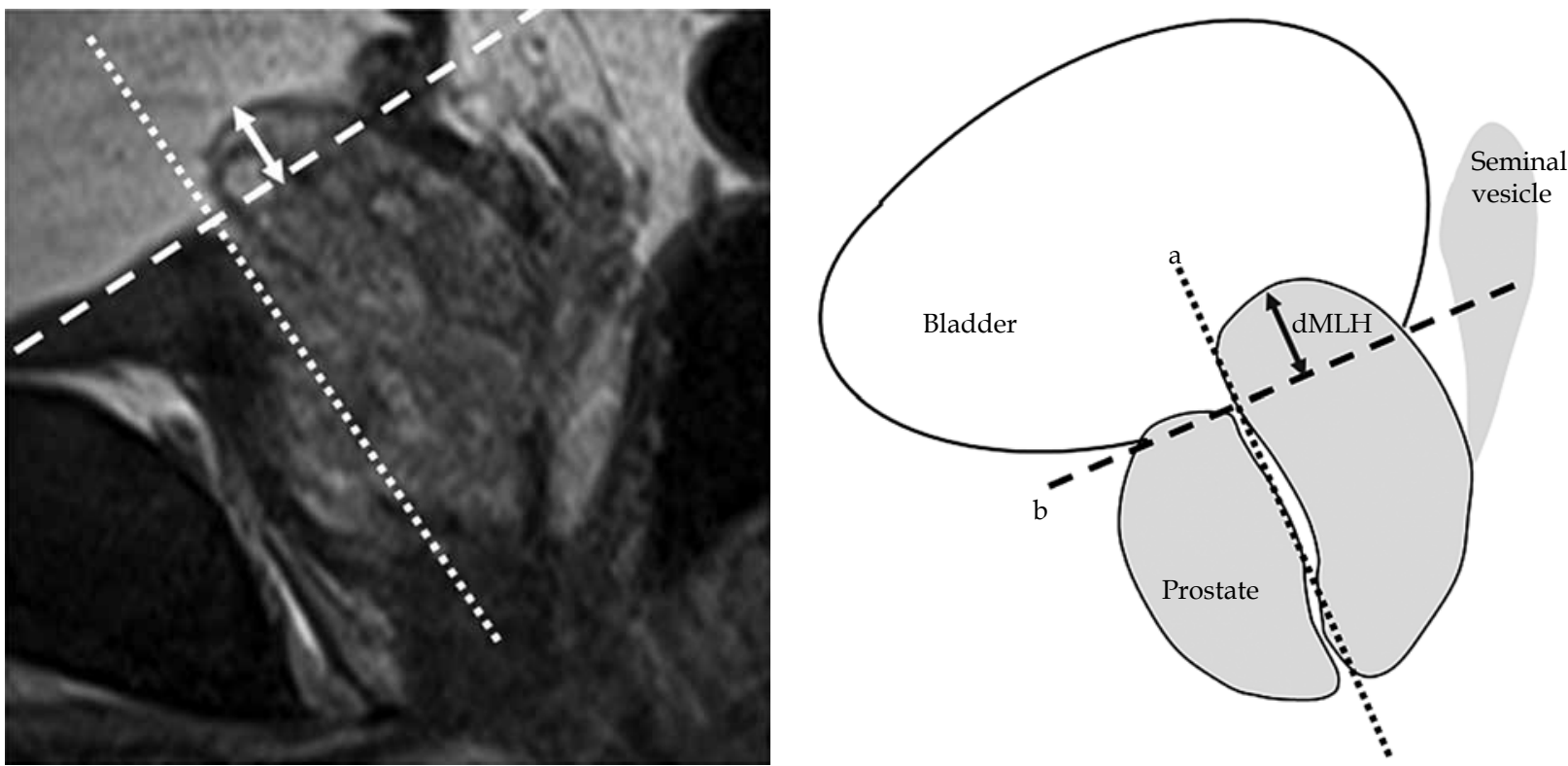

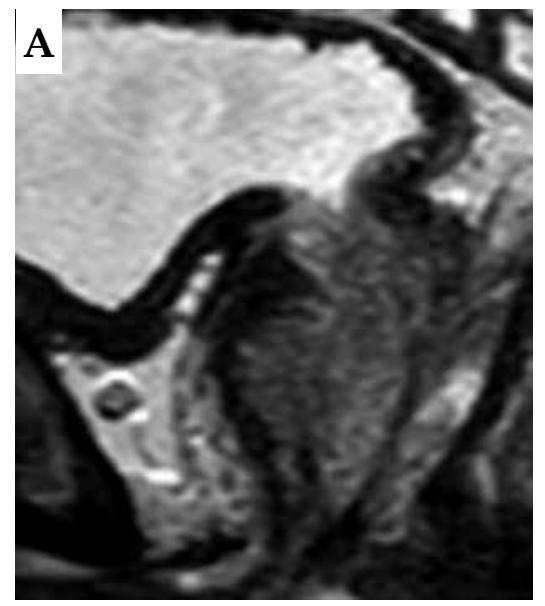

Non-MLH

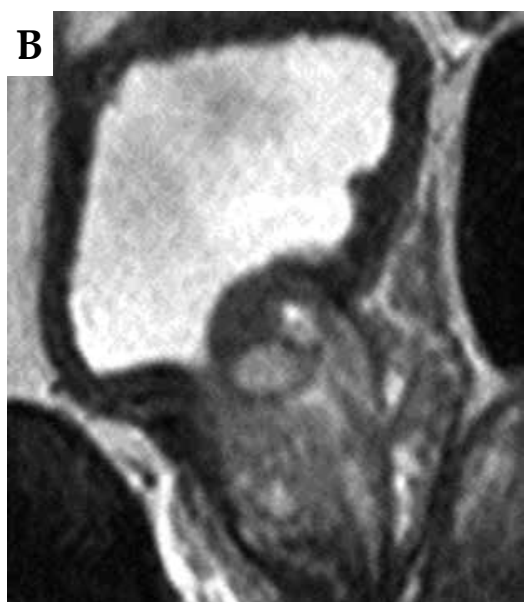

Mild $(1 \leq \mathrm{dMLH}<10 \mathrm{~mm})$

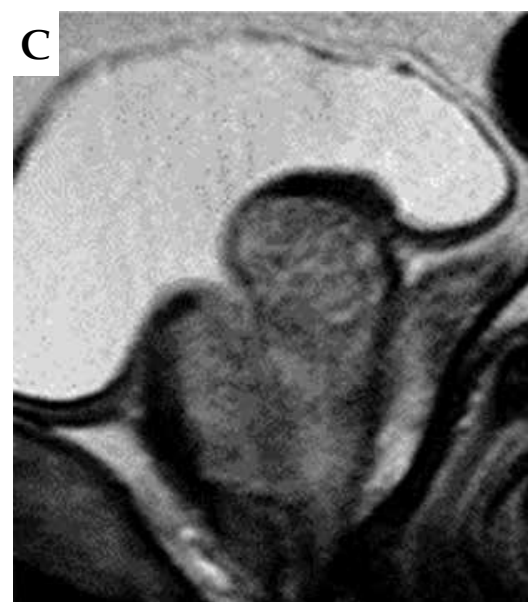

Severe $(\geq 10 \mathrm{~mm})$

Fig. 1. The distance of distance of median lobe hyperplasia (dMLH). MRI images of the prostate were obtained at $0.5 \mathrm{~cm}$ intervals with patients in the supine position. Base line: line $a$ is drawn through the long axis of the prostate, with line $b$ perpendicular and tangent to the anterior portion of the prostate (dotted line). Measuring the distance, the posterior prostatic tissue protrudes above base line (solid arrow). The classification of MLH on MRI. A) Non-MLH, B) mild MLH (dMLH is less than $10 \mathrm{~mm}$ ), C) severe MLH (dMLH is greater than or equal to $10 \mathrm{~mm}$ ) 
At our institution, MRI before LDR-BT is always performed. Sagittal MRI of the prostate was obtained at $0.5 \mathrm{~cm}$ intervals with the patients in a supine position. Among the patients encountered between 2007 and 2016, 225 patients with prostate cancer underwent LDR-BT. In total, $32(14.2 \%)$ patients with MLH identified radiologically on MRI scans and the remaining 193 non-MLH patients were analyzed.

We determined the dMLH as follows: the base line was located perpendicularly to the plane tangent to the anterior portion of the prostate (dotted line). The distance, by which the posterior prostatic tissue protruded above the base line was then measured (solid arrow) (Figure 1).

It was difficult to determine the prescribed radiation dose of dMLH in non-MLH cases, as there was no protrusion into the bladder in these patients. Therefore, we considered that the base of the prostate in non-MLH patients was located in the region of the prostate that was closest to the bladder, which was named the "near the dMLH base". We compared the prescribed radiation dose between dMLH (posterior transitional zone of prostatic tissue that protruded into the bladder) in MLH patients, and the site corresponding to the same area in non-MLH patients. The median dMLH was $5.2 \mathrm{~mm}$ (range, $2.8-9 \mathrm{~mm}$ ) in the mild MLH cases, and $11 \mathrm{~mm}$ (range, 10-12 mm) in the severe MLH cases (overall median, $7 \mathrm{~mm}$ ). Therefore, $10 \mathrm{~mm}$ was established as the baseline for classification, based on the hypothesis described in the Purpose section. The patients were divided into three groups based on their MRI findings as follows: non-MLH, mild MLH $(\mathrm{dMLH}<10 \mathrm{~mm})$, and severe MLH $(\mathrm{dMLH} \geq 10 \mathrm{~mm})$ (Figure 1). One hundred and ninety-three non-MLH cases were used as a control group in the present study.

According to Inada's definition for migrated seeds, the seed migration was defined as a seed moving more than $1.5 \mathrm{~cm}$ away from the target, without considering the dosimetric contribution to the target. Seeds, which were misplaced in periprostatic tissues or seminal vesicles were not reported as having migrated [9]. Stabilized implants are sources that do not fit the above definition.

Two radiation oncologists and three urologists who had more than three years of experience conducted intra-operative LDR-BT planning at the same time.

\section{Brachytherapy}

Treatment planning software program with Interplant ${ }^{\circledR}$ (Centers for Medicare and Medicaid Services, Baltimore, USA) and VariSeed $^{\circledR}$ (Varian Medical Systems, California, USA) were used. All patients were intra-operatively treated with free seeds, and achieved an adequate dose to the median lobe tissue. We did not use stranded seeds (custom-linked seeds) in this study. A post-implant dosimetric analysis was carried out by computed tomography $(\mathrm{CT}), 1$ month after the treatment.

The evaluation criteria were as follows: DVH for the prostate, urethra, and rectum were constructed to determine the minimal dose received by $90 \%$ of the prostate (prostate $\mathrm{D}_{90}$ ), the volume of the prostate receiving 100\% of the prescribed dose (prostate $V_{100}$ ), the minimal dose received by $5 \%$ of the urethra (urethral $D_{5}$ ), the volume of the urethra receiving $150 \%$ of the prescribed dose (urethral $\mathrm{V}_{150}$ ), and the volume of the rectum receiving $100 \%$ of the prescribed dose (rectum $\mathrm{V}_{100}$ ).

The prescribed dose was 145 Gy for brachytherapy alone, and 110 Gy for a combination therapy with a subsequent boost of 45 Gy by external beam radiotherapy (EBRT).

The patients with a high-volume prostate $(\geq 40 \mathrm{ml})$ initially underwent androgen deprivation therapy for 3-6 months, using a luteinizing hormone-releasing agonist. High-risk patients, according to the NCCN classification, received hormone therapy with combined androgen blockade (CAB) before and after brachytherapy.

\section{Progress observation/evaluation of adverse events}

Biochemical failure after brachytherapy was defined according to the ASTRO Phoenix definition $(2 \mathrm{ng} / \mathrm{ml}$ increase in prostate specific antigen [PSA] values above the nadir). Post-brachytherapy observations were carried out, measuring the PSA level every 3 months for the first 2 years and then at 6-month intervals.

Urinary obstructive symptoms were calculated using the International Prostate Symptom Score (IPSS) criteria. All cases underwent a pre-treatment and at least three follow-up IPSS evaluations. The follow-up survey was conducted at 1-, 6-, and 12-months after the treatment.

\section{Statistical analyses}

$P$ values of $<0.05$ were considered statistically significant. Categorical variables were compared using Fisher's exact test, and comparisons of continuous variables were performed using an analysis of variance or the Kruskal-Wallis test. Comparisons between groups were done using Mann-Whitney $U$-test with Bonferroni's correction. Any correlation in the prostatic volume, dMLH, and IPSS was evaluated by Spearman's rank test. IBM SPSS statistics version 23 was used for the analysis, and SAS9.4 and JMP Pro 13.1 were used to analyze IPSS data.

\section{Results}

\section{Patients' characteristics}

The patients' characteristics did not differ to a statistically significant extent, with the exception of the median follow-up, ultrasound volume, and seed number. The details of all cases are shown in Table 1 . There were significant differences in the values of urethral $\mathrm{D}_{5}$, urethral $\mathrm{V}_{150}$, seed migration, and operation time between study arms (urethral $\mathrm{D}_{5}, p=0.012$, urethral $\mathrm{V}_{150}, p=0.006$, seed migration, $p=0.009$, operation time, $p=0.0008$ ). There were significant differences in the seed migration, operation time, and coverage of MLH/base between the severe and non-severe MLH cases (seed migration, $p=0.007$, operation time, $p=0.0002$, coverage of MLH/base, $p<0.001$ ) (Table 2).

We found no significant association between the prostatic volume and dMLH or the pre-IPSS and dMLH. In contrast, a positive correlation was observed between the number of migrated seeds and $\mathrm{dMLH}$, and the operation time and prostatic volume (Figure 2). 
Table 1. Characteristics of patients

\begin{tabular}{|c|c|c|c|c|}
\hline & Non MLH & Mild & Severe & $P$-value \\
\hline No. of patients & 193 & 24 & 8 & \\
\hline Age (years), median (range) & $66(50-81)$ & $68.5(57-75)$ & $68.0(57-74)$ & 0.86 \\
\hline Follow-up (years), median (range) & $41(6-106)$ & $75(16-110)$ & $31.5(15-59)$ & 0.002 \\
\hline Ultrasound volume (cc) (range) & $25.7(10.2-46.5)$ & $31.4(19.6-50.2)$ & $34.4(24.7-44)$ & $<0.001$ \\
\hline Seed number (range) & $75(42-104)$ & $80(58-110)$ & $84.5(73-95)$ & 0.01 \\
\hline External beam radiation & 7 & 2 & 1 & 0.34 \\
\hline Neo-adjuvant hormones & 39 & 10 & 4 & 0.15 \\
\hline \multicolumn{5}{|l|}{ D’Amico } \\
\hline Low & 109 & 16 & 5 & \multirow[t]{3}{*}{0.44} \\
\hline Intermediate & 76 & 8 & 3 & \\
\hline High & 8 & 0 & 0 & \\
\hline \multicolumn{5}{|l|}{ Clinical stage } \\
\hline T1c & 138 & 16 & 7 & \multirow[t]{3}{*}{0.38} \\
\hline $\mathrm{T} 2 \mathrm{a} / \mathrm{T} 2 \mathrm{~b} / \mathrm{T} 2 \mathrm{c}$ & $38 / 8 / 7$ & $5 / 2 / 1$ & $1 / 0 / 0$ & \\
\hline T3a & 2 & 0 & 0 & \\
\hline \multicolumn{5}{|l|}{ PSA } \\
\hline$\leq 10$ & 171 & 20 & 6 & \multirow[t]{2}{*}{0.29} \\
\hline$>10$ & 22 & 4 & 2 & \\
\hline \multicolumn{5}{|l|}{ Gleason score } \\
\hline$<7$ & 171 & 20 & 6 & \multirow[t]{3}{*}{0.46} \\
\hline 7 & 22 & 4 & 2 & \\
\hline$>7$ & 171 & 20 & 6 & \\
\hline $\mathrm{dMLH}$, median (range) & - & $5.2(2.8-9)$ & $11(10-12)$ & $<0.001$ \\
\hline
\end{tabular}

MLH-median lobe hyperplasia

Table 2. Analysis of treatment outcome

\begin{tabular}{|c|c|c|c|c|}
\hline & \multicolumn{2}{|c|}{ Non severe } & \multirow[t]{2}{*}{ Severe } & \multirow[t]{2}{*}{$P$-value } \\
\hline & Non MLH & Mild & & \\
\hline No. of patient $(N=217)$ & 193 & 24 & 8 & \\
\hline Prostate $\mathrm{D}_{90}(\%)$ & 120 & 120 & 116.1 & 0.595 \\
\hline Prostate $\mathrm{V}_{100}(\%)$ & 96.5 & 96.3 & 94.7 & 0.528 \\
\hline Urethral $D_{5}(\%)$ & 166.3 & 148.8 & 175.2 & 0.674 \\
\hline Urethral $V_{150}(\%)$ & 35.4 & 6.6 & 25.1 & 0.288 \\
\hline Rectum $\mathrm{V}_{100}(\mathrm{cc})$ & 0.18 & 0.10 & 0.11 & 0.583 \\
\hline Bladder neck $\mathrm{D}_{2 c c}(\%)$ & & & 54.1 (range, 35.5-83) & \\
\hline $\begin{array}{l}\text { Seed migration (\%) } \\
\text { (migrated or misplaced } \\
\text { source/total) }\end{array}$ & $31.6(61 / 193)$ & $16.7(4 / 24)$ & $75(6 / 8)$ & 0.007 \\
\hline Operation time (min) & 80 & 89.5 & 135.0 & 0.0002 \\
\hline $\begin{array}{l}\text { Coverage of base/MLH } \\
(\%)(\text { cold spot/total) }\end{array}$ & $0.02(4 / 193)$ & $20.8(5 / 24)$ & $62.5(5 / 8)$ & $<0.001$ \\
\hline Recurrence & $\begin{array}{c}\text { PSA } 6 \\
\text { Metastasis 2: } \\
\text { Lymph node } 1 \\
\text { Bone } 1\end{array}$ & 0 & 0 & \\
\hline
\end{tabular}


A

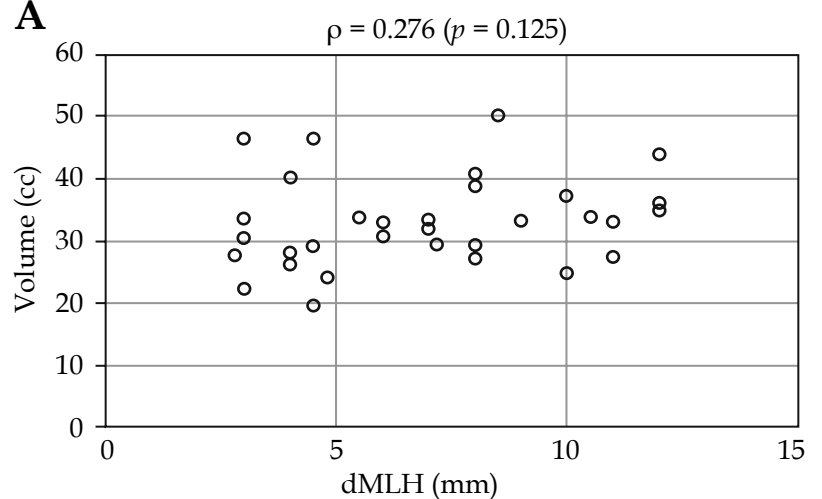

C

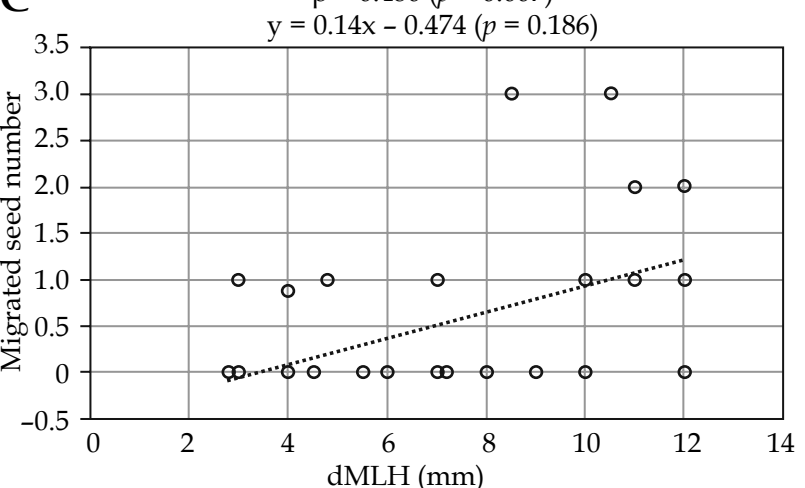

B

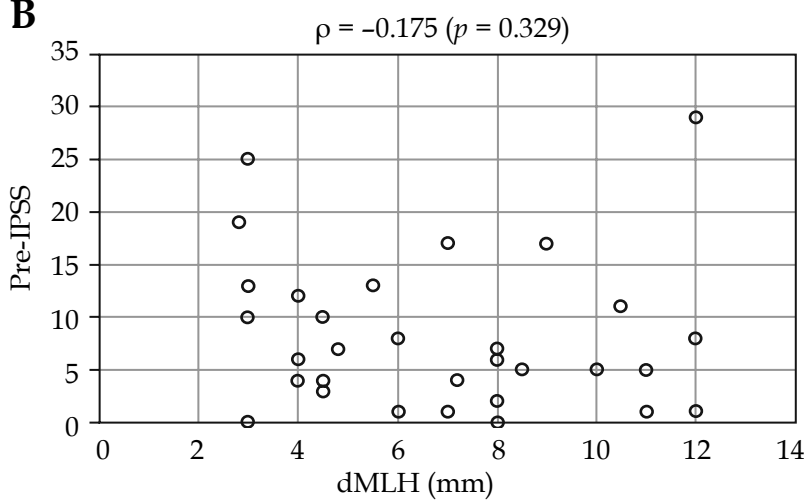

D

$\rho=0.371(p=0.038)$

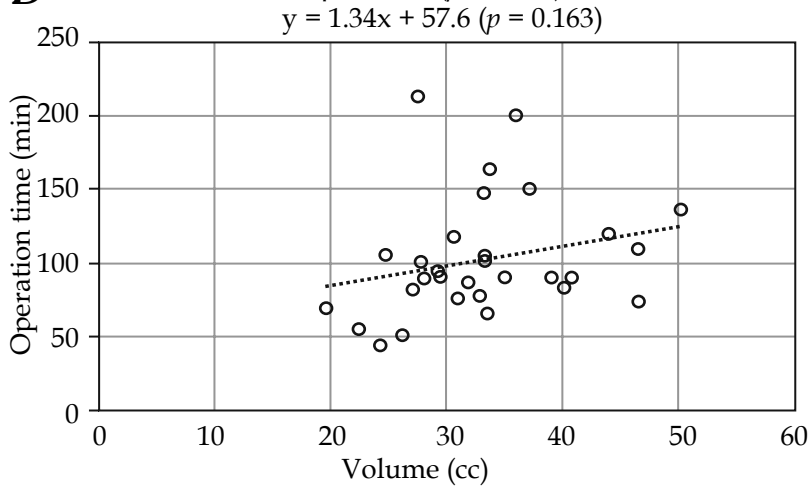

Fig. 2. Spearman's rank correlation coefficient. A) No correlation between prostatic volume and distance of median lobe hyperplasia (dMLH). B) No correlation between pre-IPSS and dMLH. C) A weak correlation between migrated seed number and dMLH. D) A weak correlation between operation time and prostatic volume

\section{Recurrent outcome}

Biochemical and clinical recurrence were detected in 7 non-MLH patients, including PSA failure in 6 and lymph node and bone metastasis in 1 . None of the cases in the mild and severe MLH groups had biochemical failure or local recurrence (Table 2).

\section{$D V H$}

The post-implant prescription isodose covered 89-99\% of the target volume. The implant dosimetry calculation (DVH: prostate $\mathrm{D}_{90}$, prostate $\mathrm{V}_{100}$, and rectum $\mathrm{V}_{100}$ ) did not differ significantly among patients with non-MLH, mild MLH, and severe MLH $(p=0.63, p=0.81$, and $p=0.30$, respectively; Table 2). There was also no significant difference in the post-planning dose delivered to the prostate $\left(D_{90}, V_{100}\right)$ among the three groups, regardless of the possibility of seed migration. In this study, 6 of the 8 patients with severe MLH presented seed migration, and a cold spot at the IPP occurred in 5 cases. According to Hathout's definition, the bladder neck was defined as $5 \mathrm{~mm}$ around Foley catheter, which defined urethra on CT slices between the catheter balloon and the prostatic urethra to a minimum volume of $2 \mathrm{~cm}^{3}$ (median, $2.2 \mathrm{~cm}^{3}$ ) [14]. The bladder neck $D_{2 c c}$ was $>50 \%$ in 5 of 8 cases, with a median of $54 \%$.

\section{Seed migration}

Seed migration occurred in $10(31.3 \%)$ of the $32 \mathrm{MLH}$ patients. In $6(60 \%)$ of the 10 patients, only a single seed mi- grated. Sixty-one patients $(31.6 \%)$ in the non-MLH group showed seed migration. Two, three, and four migrated seeds were observed in 10,10, and 3 cases, respectively, out of 61 , while the remaining cases demonstrated only a single migrated seed in the non-MLH group. Three migrated seeds were observed in 3 out of 4 cases, while the remaining case demonstrated only a single migrated seed in the mild MLH group. Two and three migrated seeds were observed in 2 cases and 1 case, respectively, out of 6 , while the remaining cases demonstrated only a single migrated seed in the severe MLH group; there was no significant difference in the incidence of seed migration between the MLH and non-MLH groups. Some of the patients overlapped. The individual migration rate in non-severe and severe cases was $10.1 \%$ and $25 \%$, respectively, in the lung, $6.8 \%$ and $0 \%$, respectively, in the seminal vesicle, $12.4 \%$ and $37.5 \%$, respectively, in the pelvis, and $6.0 \%$ and $25 \%$, respectively, in the bladder; the other distant migration rates were very small in each group. However, implant migration $(\mathrm{OR}=7$, $p=0.03)$ and low-dose area of the median lobe (OR $=38.5$, $p<0.0001)$ tended to be higher in the severe MLH group. There was a significant difference between the non-severe and severe MLH groups in the coverage of the region of the prostate closest to the bladder. Although there was no significant difference in coverage between the mild and severe MLH groups, the coverage tended to be lower in the severe MLH group.

We divided the dMLH into four groups by quartiles, and seed migration into three groups. We performed the 
Table 3. Jonckheere-Terpstra test for relationships of migrated seed and distance of median lobe hyperplasia $(\mathrm{dMLH})$

\begin{tabular}{lcccc}
$\mathrm{d}$ MLH (quartile group) & Migrated seed $=0$ & Migrated seed $=1$ & Migrated seed $=2,3$ & Total \\
\hline $1(\mathrm{dMLH}<4.125)$ & $7(87.5 \%)$ & $1(12.5 \%)$ & $0(0 \%)$ & 8 \\
\hline $2(4.125 \leq \mathrm{dMLH}<7)$ & $6(85.71 \%)$ & $1(14.29 \%)$ & $0(0 \%)$ & 7 \\
\hline $3(7 \leq \mathrm{dMLH}<9.75)$ & $7(77.78 \%)$ & $1(11.11 \%)$ & $1(11.11 \%)$ & 9 \\
\hline $4(\mathrm{dMLH} \geq 9.75)$ & $2(25 \%)$ & $3(37.5 \%)$ & $3(37.5 \%)$ & 8 \\
\hline Total & 22 & 6 & 4 & 32
\end{tabular}

MLH-median lobe hyperplasia

Jonckheere-Terpstra trend test, which revealed a tendency for seed migration to increase, as the value of $\mathrm{dMLH}$ increased $(p=0.0049)$ (Table 3).

\section{Adverse events}

The chronological changes in the IPSS (pre-treatment, after one month, and then at six-month intervals) are shown in Figure 3. Generally, we found that the IPSS peaked at one month after implantation, resolving slowly and returning to the pre-treatment level within one year. A mixed-effects model was used to analyze the time-trend of IPSS between the non-severe and severe MLH groups. The time group interaction was significant $(p=0.0155)$. This suggests that there was a significant difference between the two groups in the time-trend of IPSS. The change of IPSS between baseline and one month, between one month and 6 months, and between one month and 12 months, was compared among the two groups. Figure 3 shows a "least-square means plot (predicted means)". The group difference at each timepoint was also tested. The IPSS in the severe group was significantly higher than that in the non-severe group $(p=0.008)$. This analysis showed a significant correlation between time and chronological changes in the IPSS $(p<0.0001)$ (Figure 3). Among the patients with severe MLH, grade 2 urinary retention was observed in 1 case, grade 3 urinary retention was observed in 2 cases, and grade 2 hematuria was observed in 1 patient as adverse events.

Regarding late adverse events after two years, in the MLH group, grade 2 urinary toxicity (urinary retention, urinary tract pain, prostatic hemorrhage, and erectile dysfunction) occurred in 4 patients (12\%), and grade 3 (hematuria) urinary toxicity occurred in 1 case $(3 \%)$. There was not much difference in the incidence of acute and late dysuria between the cases of severe and non-severe MLH.

\section{Discussion}

Median lobe hyperplasia is defined as the protrusion of hypertrophied prostate tissue into the bladder. MLH is derived from periurethral tissue, and protrusion correlates with urinary obstruction rather than the prostate volume [2]. Although no definitive conclusions have been made, an intravesical prostatic protrusion is believed to be caused by enlarging lateral lobes and median lobe; the protrusion of the prostate then causes ball-valve-type obstruction, disrupting the funneling effect of the bladder

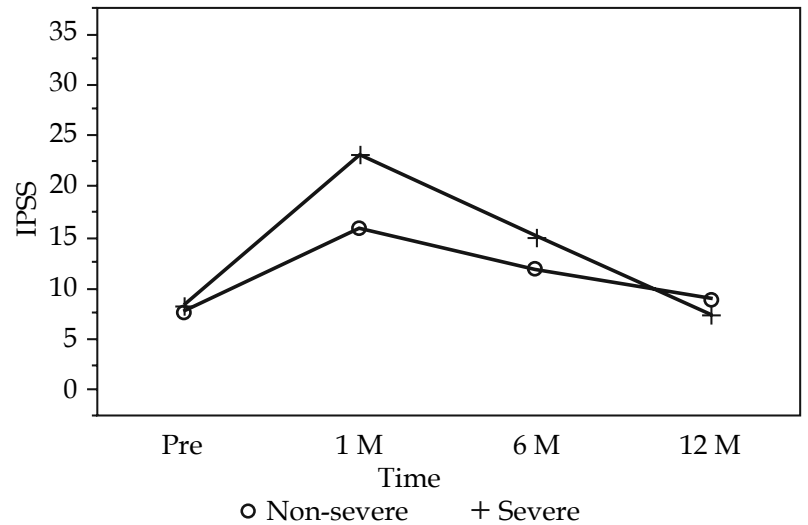

Fig. 3. Change of IPSS between baseline and one month, between one month and 6 month, and between one month and 12 months, were compared among non-severe and severe median lobe hyperplasia (MLH) groups

neck and causing dyskinetic movement of the bladder during voiding [10].

The ABS Prostate Low-Dose-Rate Task Group suggests that severe MLH is a relative contraindication for brachytherapy. On the other hand, we consider the reasons for this to be technical difficulties, which are associated with inadequate dose coverage of the protruded median lobe, and the risk of migration and urinary retention [1]. However, the threshold levels of MLH as a contraindication are not clearly described in the ABS criteria, and the literature on prostate brachytherapy with MLH is limited.

\section{Usefulness of MRI for assessment}

Wallner and Nguyen previously reported that MLH did not appear to be a strong contraindication for prostate brachytherapy, and that prophylactic resection of hypertrophic tissue in such patients was probably not warranted $[8,11]$. They measured the dMLH using TRUS; however, this method may be associated with measurement errors due to heterogeneous technical skills in the performance of ultrasonography. In the present study, we evaluated the dMLH objectively using sagittal MRI, as Wallner did with TRUS.

\section{Seed migration}

Severe MLH with inadequate dose coverage of the protruded median lobe might be closely related to anatomically unstable implanted seeds. 
There is no standard definition of a cold spot area. To evaluate the cold spot area, we applied Morén's definition [12]. They defined a cold spot as a contiguous volume that receives less than $90 \%$ of the prescribed dose.

It may be possible to cover cold spots of MLH caused by seed migration instead of other techniques, for example, intensity modulated radiation therapy (IMRT), including simultaneous integrated boost (SIB) technique and other different types of seeds, such as connecting seeds (stranded seeds) and ${ }^{125}$ I sources coated with polyglactin 910 suture (TheraStrand-SL ${ }^{\circledR}$ ) [13].

Although the region of the prostate closest to the bladder tended to have a lower dose area in the MLH group, there was no significant difference in the treatment outcomes of post-plan DVH or seed migration between the MLH and non-MLH groups.

A few migrated seeds had an insignificant effect on the isodose coverage of the base area. The reason was that we intentionally inserted excessive seeds into the area, in which the prostate tissue protruded into the bladder.

\section{DVH}

DVH did not change, despite the possibility of migrated seeds. The coverage of MLH decreased significantly in cases of severe MLH. Nevertheless, the $D_{90}$ for the prostate decreased to $4 \%$; the $\mathrm{V}_{100}$ for the prostate decreased $1-2 \%$ in the severe MLH group comparing to the non-severe MLH group. There was a low-dose area in patients with MLH. However, the DVH parameters, such as $D_{90}$ and $V_{100}$ for the prostate were not significantly reduced.

There were significant differences in urethral dose. The $D_{5}$ and $V_{150}$ for the urethra was significantly higher in the non-MLH group than in the mild MLH group, which was contrary to our expectations. We assumed that the prostatic volume had an effect on the urethral dose, because the prostatic volume of the non-MLH patients was smaller in comparison to the MLH patients. To avoid post-operative urinary retention, we may intentionally optimize planning and implant seeds in the prostate with a much lower urethral dose.

\section{Adverse events}

The present study showed no specific tendency regarding genitourinary toxicity in the patients with severe or non-severe MLH. It is difficult to establish whether or not dMLH and dysuria were relevant to these outcomes. The maximum dMLH was approximately $12 \mathrm{~mm}$ in severe MLH cases. The reason why there was no significant difference in urination disorder was due to our exclusion of patients with severe dysuria and very severe dMLH (e.g., > $20 \mathrm{~mm}$ in size), as those patients were treated with transurethral resection of the prostate (TURP) or HoLEP.

\section{Operation time}

There was a positive correlation between the operation time and prostate volume.

\section{Bladder neck sparing}

The bladder neck preservation brachytherapy in a MLH patient may be technically difficult. The reasons are summarized in the following two hypotheses. Radioactive seeds implanted into the intravesical prostatic protrusion are predisposed to migration after brachytherapy. Due to the bearing of IPP on the bladder neck funneling, the use of radioactive implants close to the bladder neck is considered to be a risk factor for urinary retention.

Hathout et al. reported that bladder neck $\mathrm{D}_{2 \mathrm{cc}}>50 \%$ was the strongest predictor of grade 2 acute and late urinary toxicity in patients treated with LDR-BT [14]. In this study, 6 of the 8 patients with severe MLH presented seed migration, and a cold spot at the IPP occurred in 5 cases. The bladder neck $D_{2 c c}$ was $>50 \%$ in 5 of 8 cases, with a median of $54 \%$. However, the post-implant course was uneventful, without local recurrence at the IPP. There was not much difference in the rates of acute and late dysuria between the patients with severe and non-severe MLH (Table 4).

The bladder neck constraints in brachytherapy planning may be helpful for reducing urinary toxicity. However, the bladder neck dose did not significantly increase the degree of dysuria in our study, and thus, no consensus was reached on how to contour and to constrain the bladder neck.

In the case of a focal lesion that is not located near to the bladder neck, if we evaluate the volume of the intravesical prostatic protrusion and bladder neck dose in the pre-planning phase, and investigate whether stable seed implantation is possible using sources coated with polyglactin 910 suture (Theragenics AgX100® I-125 seeds) and custom-linked seeds, it might be possible to perform bladder neck sparing brachytherapy. In cases, in which source implantation is associated with technical difficulty, bladder neck sparing robot-assisted laparoscopic radical prostatectomy (RALP) represents an effective tool for treating a recurrence that is not located near the bladder neck [15].

\section{Conclusions}

In the case of $\mathrm{dMLH}<10 \mathrm{~mm}$ on sagittal MRI, if we evaluate the volume of intravesical prostatic protrusion and bladder neck dose in the pre-planning phase, and investigate whether stable seed implantation is possible using sources coated with polyglactin 910 suture (Theragenics $\mathrm{AgX100®} \mathrm{I-125} \mathrm{seeds)} \mathrm{and} \mathrm{custom-linked} \mathrm{seeds,}$ MLH does not appear to be a strong contraindication for LDR-BT. However, seed migration and cold spots tended to be more frequent in severe MLH exceeding $\mathrm{dMLH}$ $10 \mathrm{~mm}$; thus, attention is required when treating patients with severe MLH. The adaptation of the method to evaluate dMLH on sagittal MRI would be an effective option for implementing LDR-BT for cases with MLH.

To curb rising medical costs, MRI is recommended when MLH is suspected based on TRUS during a prostate biopsy. Future studies with larger cohorts are needed to investigate long-term follow-up data. 


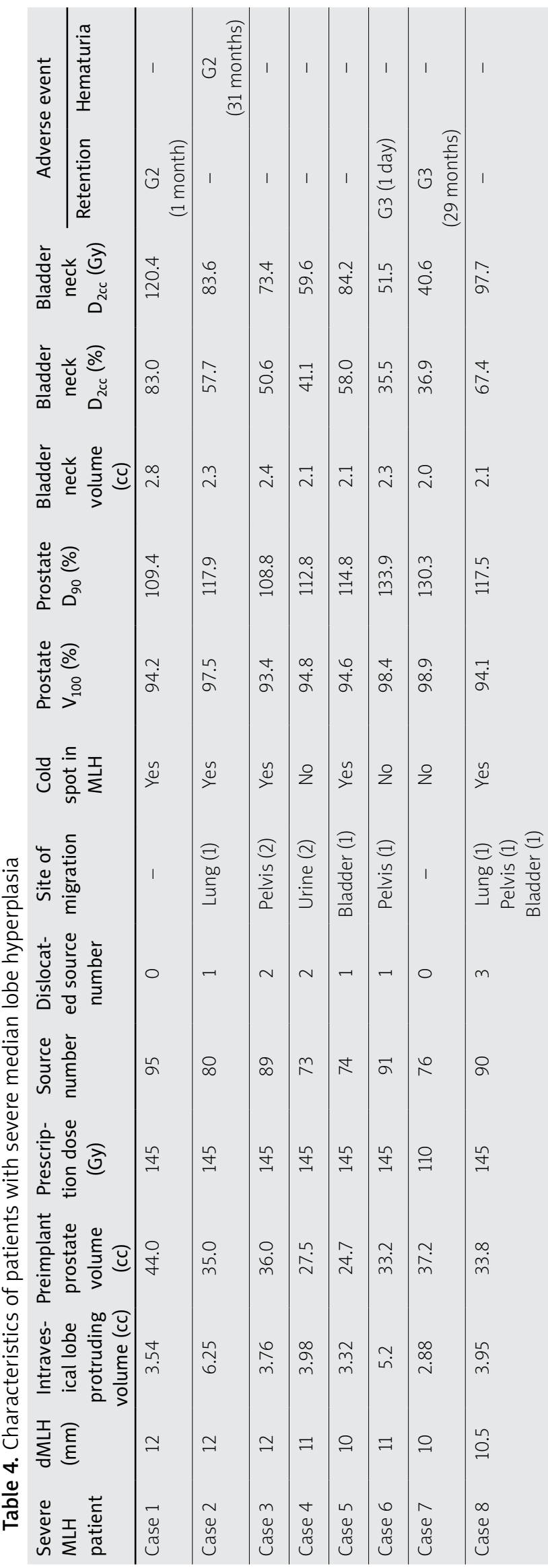

\section{Limitations}

The present study was associated with some limitations. First, our findings should be interpreted with caution due to uncertainties in the following respect. The present study was limited by its inclusion of cases without very severe dysuria and dMLH exceeding $20 \mathrm{~mm}$. Moreover, the small sample sizes of severe MLH are biased in the first half of $10 \mathrm{~mm}$ that severe MLH population presented with dMLH between 10 and $12 \mathrm{~mm}$. Second, this was a single-institution study. Third, because there were only eight severe MLH cases, we were unable to make an objective assessment. Fourth, we cannot discount the possibility of a measurement error in the evaluations of the $\mathrm{dMLH}$ in this study. Fifth, the number of implant sources placed on the IPP was increased in severe MLH cases in this study. We intentionally implanted more sources on the IPP because of the fear of seed migration in the cases of severe MLH. Without seed migration, these patients would be at greater risk to receive a high-dose at the bladder neck in comparison to cases with migration. Finally, the surgeon and radiation treatment planner were not always the same. Further study is required to examine the validity of threshold with a higher number of patients.

\section{Acknowledgments}

The authors report no proprietary or commercial interest in any product mentioned or concept discussed in this article. This research did not receive any specific grant from funding agencies of the public, commercial, or not-for-profit sectors.

\section{Disclosure}

The authors report no conflict of interest.

\section{References}

1. Davis BJ, Horwitz EM, Lee WR et al. American Brachytherapy Society consensus guidelines for transrectal ultrasound-guided permanent prostate brachytherapy. Brachytherapy 2012; 11: 6-19.

2. Nose H, Foo KT, Lim KB et al. Accuracy of two noninvasive methods of diagnosing bladder outlet obstruction using ultrasonography: intravesical prostatic protrusion and velocity-flow video urodynamics. Urology 2005; 65: 493-497.

3. Chia SJ, Heng CT, Chan SP et al. Correlation of intravesical prostatic protrusion with bladder outlet obstruction. BJU Int 2003; 91: 371-374.

4. Chung SD, Chiu B, Yu H et al. Re: Keqin Z et al. Clinical significance of intravesical prostatic protrusion in patients with benign prostatic enlargement. (Urology 2007;70:1096-1099). Urology 2009; 73: 216.

5. Park HY, Lee JY, Park SY et al. Efficacy of alpha blocker treatment according to the degree of intravesical prostatic protrusion detected by transrectal ultrasonography in patients with benign prostatic hyperplasia. Korean J Urol 2012; 53: 92-97.

6. Keqin Z, Zhishun X, Jing Z et al. Clinical significance of intravesical prostatic protrusion in patients with benign prostatic enlargement. Urology 2007; 70: 1096-1099.

7. Malde S, Nambiar AK, Umbach R et al. Systematic review of the performance of noninvasive tests in diagnosing bladder outlet obstruction in men with lower urinary tract symptoms. Eur Urol 2017; 71: 391-402. 
8. Wallner K, Smathers S, Sutlief $S$ et al. Prostate brachytherapy in patients with median lobe hyperplasia. Int J Cancer 2000; 90: 152-156.

9. Inada M, Yokokawa M, Minami T et al. Dosimetry advantages of intraoperatively built custom-linked seeds compared with loose seeds in permanent prostate brachytherapy. J Contemp Brachytherapy 2017; 9: 410-417.

10. Chia SJ, Heng CT, Chan SP et al. Correlation of intravesical prostatic protrusion with bladder outlet obstruction. BJU Int 2003; 91: 371-374.

11. Nguyen J, Wallner K, Han B et al. Urinary morbidity in brachytherapy patients with median lobe hyperplasia. Brachytherapy 2002; 1: 42-47.

12. Morén B, Larsson T, Tedgren ÅC et al. A mathematical optimization model for spatial adjustments of dose distributions in high dose-rate brachytherapy. Phys Med Biol 2019; 22: 225012.

13. Reed DR, Wallner KE, Merrick GS et al. A prospective randomized comparison of stranded vs. loose 125I seeds for prostate brachytherapy. Brachytherapy 2007; 6: 129-134.

14. Hathout L, Folkert MR, Kollmeier MA et al. Dose to the bladder neck is the most important predictor for acute and late toxicity after low-dose-rate prostate brachytherapy: implications for establishing new dose constraints for treatment planning. Int J Radiat Oncol Biol Phys 2014; 90: 312-319.

15. Preisser F, Martin LB, Pompe RS et al. Effect of bladder neck sparing at robot-assisted laparoscopic prostatectomy on postoperative continence rates and biochemical recurrence. Urol Oncol 2020; 38: 1.e11-1.e16. 